\title{
Walking and Crawling with ALoF A Robot for Autonomous Locomotion on Four Legs
}

\section{Conference Paper}

\section{Author(s):}

Remy, C.David; Baur, Oliver; Latta, Martin; Lauber, Andi; Hutter, Marco (1); Hoepflinger, Mark A.; Pradalier, Cédric; Siegwart, Roland

\section{Publication date:}

2010

Permanent link:

https://doi.org/10.3929/ethz-a-010185444

Rights / license:

In Copyright - Non-Commercial Use Permitted 


\title{
WALKING AND CRAWLING WITH ALOF - A ROBOT FOR AUTONOMOUS LOCOMOTION ON FOUR LEGS*
}

\author{
C. DAVID REMY, OLIVER BAUR, MARTIN LATTA, ANDI LAUBER, MARCO \\ HUTTER, MARK HOEPFLINGER, CÉDRIC PRADALIER, ROLAND SIEGWART \\ Autonomous Systems Lab, Institute of Robotics and Intelligent Systems, Swiss Federal \\ Institute of Technology (ETHZ), Zurich, Switzerland
}

\begin{abstract}
In this paper we introduce the robotic platform ALoF that is designed to aid research on perception in legged locomotion. Well balanced size and complexity results in a robust platform that is easy to handle, yet able to perform complex maneuvers as well as carry more sophisticated 3D sensors. A very large range of motion allows the robot to actively explore its surroundings through haptic interaction, and to choose between a wider range of planning options. This robot was employed and tested in the lunar robotics challenge, for which we also developed a novel crawling gait, in which the weight of the robot is alternately supported by scaled plates under the main body and the four shank segments. This allowed for stable locomotion in steep terrain with very loose soil.
\end{abstract}

\section{Introduction}

The advantages of legs, in comparison to other principles of locomotion, are in large part confined to difficult and rough terrain in which other systems might fail completely. For most other scenarios, alternative systems generally show a superior performance, especially with respect to locomotion speed and energy consumption, - -the two factors that largely limit the operational range of mobile robots and hence their physical autonomy. While research on the principles of legged locomotion is highly desirable to lessen these disadvantages, the ultimate goal of legged locomotion systems must be the application of these systems in rough and highly unstructured terrain. Recent findings have shown impressively, that this is possible with the current state of the art. For example, in the DARPA learning locomotion challenge $[1,2]$. Yet, the robots in this project were at all times made aware of their exact state and the detailed shape and properties of the surrounding terrain, thereby reducing the task of locomotion to a problem of planning and execution. In any given real application, however, such detailed

\footnotetext{
* This work was supported in part by the Swiss National Science Foundation (SNF) (project 200021_119965/1).
} 
knowledge is not readily available. It must be collected by the robot itself, thereby generating an additional task for perception.

The intention of this paper is twofold: On the one hand, it serves the purpose of describing a newly developed research platform ALoF that is designed to aid research on perception for legged locomotion and foster the creation on systems that are able to navigate fully autonomously through rough terrain [3]. In the second part, we describe some extensions we added to this system during the Lunar Robotics Challenge (LRC) in the fall of 2008 and share the experiences we gathered there. Special attention is thereby paid to a novel crawling gait that we developed for locomotion in steep and sandy terrain.

\section{System Description}

Our robot (fig. 1) is a four legged quadruped with a total weight of about $15 \mathrm{Kg}$ and linear dimensions in the range of half a meter. This means the platform is small enough to be handled by one person alone, yet able to carry larger and more sophisticated sensors, as for example stereo cameras, laser range finders, and the like. Each of the legs has three degrees of freedom, allowing for hip abduction/adduction, hip flexion/extension, as well as knee flexion/extension. The three joints are set up in a 'mamalian' configuration, with the two knees facing each other, which generates more symmetrical ground contact forces. The feet are not actuated. We limited ourselves to the minimal number of degrees of freedom, to keep the complexity low and thus increase the robustness, modularity, and ease of maintenance of the system. This is particularly desirable for a scientific platform where things are bound to go wrong.

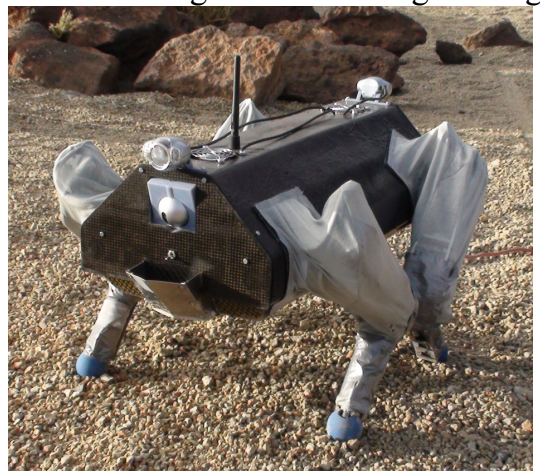

Fig. 1. The $A L o F$ robot during the ESA Lunar Robotics Challenge. In addition to its basic configuration it was equipped with pan-tilt cameras, lights, a dust-protection cover, and scaled plates under the shanks and the main body.

A bevel gear drive at the knee allows for a large motion of the knee joint, which can flex $160 \mathrm{deg}$ and extend 90 deg from a fully outstretched position. 
The two hip joints are actuated by a differential drive system that allows a 360 deg rotation of the leg about the flexion/extension-axis (the actual range of motion is solely limited by the need for a power and signal connection to the knee-joint). The main body was specifically designed to impede hip abduction/adduction as little as possible. This motion can therefore reach \pm 45 deg from vertically downwards (fig 2). Such a large range of motion allows for a greater variation of foot placement and hence provides the robot with the necessary choices for challenging planning tasks. It facilitates haptic exploration of the terrain, and enables the execution of alternative gait patterns recovery maneuvers. This includes, for example, the task to stand up after falling down, which adds an intrinsic robustness component to the locomotion task.

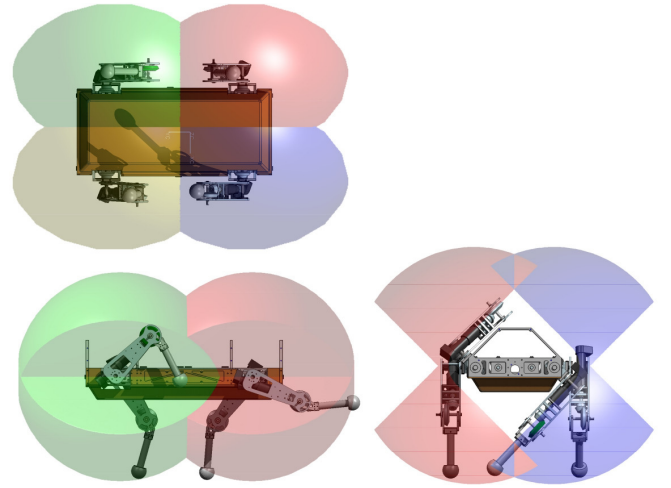

Fig. 2. A differential drive at the hip, bevel gears at the knee, and the compact design of the main body allow a very large range of motion for all legs.

To be able to actually exploit this large range of motion, we equipped all joints with strong actuators. 12 DC motors (Maxon RE25, 24V) with a gear-box ratio of 79:1, (which is additionally amplified by the bevel-gears) generate enough torque to lift about four times the robot weight when standing up from the ground. 12 individual servo controllers connected by a CAN-bus take care of low-level velocity/position control and are controlled by a NI single-board RIO, which combines a FPGA-controller and a real-time processor for the different layers of data acquisition and control. This controller is connected to a host-computer via a UDP/IP connection. High level control and energy-supply are provided off-board to save weight and reduce the complexity. The detailed hardware characteristics are listed in table 1.

\section{ALoF at the Lunar Robotics Challenge}

The goal of the Lunar Robotics Challenge (LRC) [4] was to evaluate different locomotion systems that were able to collect soil specimens in a simulated lunar 
crater and return these samples to a lander module. With regard to locomotion, the main challenge lay in the very steep terrain of the crater, which was additionally covered with loose soil, rendering standard wheeled locomotion systems useless. The teams had additional to cope with challenging lighting conditions and the missing line-of-sight between the lander and the bottom of the crater. This made navigation, control, and communication very difficult. Table 1. Hardware characteristics of the ALoF robot.

\begin{tabular}{|c|c|c|c|}
\hline Parameter & Value & Parameter (cont.) & Value \\
\hline Overall Length & $557 \mathrm{~mm}$ & $\begin{array}{l}\text { Spacing between hip joints } \\
\text { (longitudinal) }\end{array}$ & $389 \mathrm{~mm}$ \\
\hline Typical Height & $400 \mathrm{~mm}$ & Spacing between hip joints (lateral) & $208 \mathrm{~mm}$ \\
\hline Overall Width & $386 \mathrm{~mm}$ & Length of Thigh Segment & $150 \mathrm{~mm}$ \\
\hline Total Mass & $15 \mathrm{Kg}$ & Length of Shank Segment & $150 \mathrm{~mm}$ \\
\hline Main Body Mass & $10 \mathrm{Kg}$ & Foot diameter & $40 \mathrm{~mm}$ \\
\hline $\begin{array}{c}\text { Torque Knee } \\
\text { (continuous/peak) }\end{array}$ & $\begin{array}{l}4 \mathrm{Nm} / \\
15 \mathrm{Nm}\end{array}$ & $\begin{array}{l}\text { Maximum peak load for standing } \\
\text { up from the ground }\end{array}$ & $400 \mathrm{~N}$ \\
\hline $\begin{array}{c}\text { Torque Hip } \\
\text { (continuous/peak) }\end{array}$ & $\begin{array}{c}3 \mathrm{Nm} / \\
11.3 \mathrm{Nm}\end{array}$ & $\begin{array}{l}\text { Range of motion for hip } \\
\text { flexion/extension }\end{array}$ & \pm 180 deg \\
\hline $\begin{array}{l}\text { Range of motion for hip } \\
\text { abduction/adduction }\end{array}$ & $\pm 45 \mathrm{deg}$ & $\begin{array}{l}\text { Range of motion for knee } \\
\text { flexion/extension }\end{array}$ & $\begin{array}{l}+90 /-160 \\
\text { deg }\end{array}$ \\
\hline
\end{tabular}

The team of ETH Zurich implemented three collaborating robots that jointly tackled this challenge. The main task of descending into the crater, collecting the sample, and returning it to the lander was performed by ALoF. Some modifications and extensions to this robot were made specifically for the challenge: It was equipped on both ends with a Pan Tilt camera and bright illumination to enable controlling via a remote operator. As, unlike in wheeled systems, no continuously rotating parts exist, it was possible to cover the entire robot in a sealed fabric (a sort of 'jumpsuit'), that excluded dust and small rocks. This only slightly impeded the motion of the legs. The communication link with the host-computer was established by a wireless connection. The robot was remote-controlled from a ground station that commanded individual steps or step-sequences.

During the entire mission, ALoF was attached to a safety cable which was deployed from a second stationary robot at the landing module. The cable was used to passively stabilize the robot on the slope, to enhance its climbing capabilities, and to supply the necessary energy. A third robot, a 6-wheeled rover, was driving directly up to the crater rim, where it was used as bridge for wireless communication. Equipped with a high resolution pan-tilt-zoom (20x) camera, this robot also provided an advantageous birds-eye view of the crater area which greatly facilitated orientation and navigation. The rover was equipped with the CRAB passive suspension system [5], that is optimized for 
locomotion in rough terrain. For communication, we relied on a two-step wireless $802.11 \mathrm{~g}$ bridge (relayed from the landing platform to the quadrupedal robot via the wheeled rover).

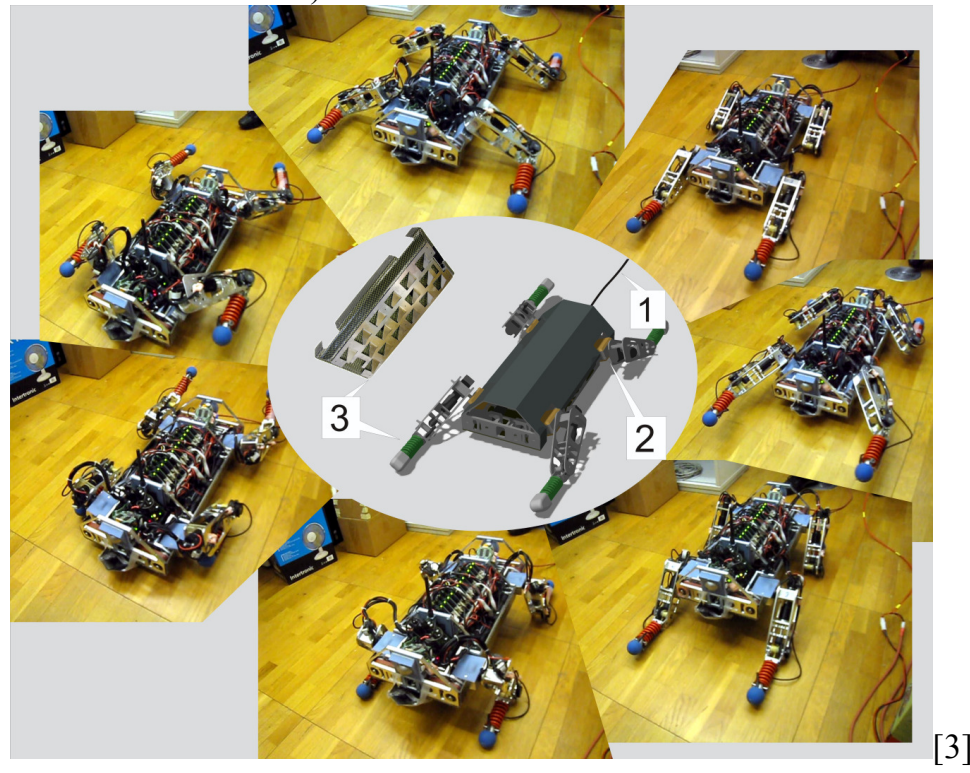

Fig. 3. The main task of sample return in the LRC was performed by ALoF, which was suspended by a tether-cable (1). The large range of motion joints at the hip (2) allowed the robot to walk upright, but also to perform a crawling motion while keeping the center of mass low. On the slopes of the crater, the crawling motion was supported by scaled plates (3) under the main body and shanks. The video stills at the outside illustrate the crawling motion (clockwise $=$ forward crawl).

In highly unfavorable terrain, such as on the slopes of the crater, due to its large range of motion, ALoF was able to lower itself onto its main body and switch from a regular static walking gait to a crawling or sliding motion (fig. 3). This allowed the robot to inch itself up or down the crater, even on extremely steep slopes. In this mode, the body weight was either supported by all four legs at once (when moving forward), or by the main body (when retracting the legs), thereby increasing the overall area of support. By equipping the robot on the bottom side of its main body and shanks with scaled plates, further measures against slipping, sliding, or caving in were introduced (fig. 4).

This crawling concept performed exceptional well in the demanding environment of the challenge. The scaled plates under the main body and shank segments dug deep into the loose soil and stably supported the robot, even in the steep slopes of the simulated crater. The only issue not fully satisfactorily solved, was lateral motion. The scales were optimized for forward motion and created only little resistance when employed laterally. This led to unwanted 
drifting when the robot crawled on lateral slopes and reduced the angular motion when turning. The drift also corrupted odometry, as the robot was turning and sliding downhill, when commanded to move in a straight line. During the challenge, it became apparent that the crawling quadruped didn't require the tethered support at all; not even on the slopes of the crater. The tethered solution was, in retrospective, an unnecessary complication.

\section{Conclusion}

This paper introduced the ALoF robot, a mid-sized research platform that is intended for the study of perception in legged locomotion. This platform was employed and tested during the Lunar Robotic Challenge, where we implemented a novel crawling gait that allowed steady locomotion on steep surfaces covered with very loose soil. While the task of the LRC was not a direct application of perception (all high-level commands were directly issued by the operator in the ground station), it was a great test to prove the robustness and usability of the robot.

\section{Acknowledgments}

The authors want to thank the European Space Agency for granting the opportunity to participate in the Lunar Robotics Challenge 2008.

\section{References}

K. Byl, K. Shkolnik, S. Prentice, N. Roy, and R. Tedrake, "Reliable Dynamic Motions for a Stiff Quadruped," Experimental Robotics, pp. 319-328, 2009.

J. R. Rebula, P. D. Neuhaus, B. V. Bonnlander, M. J. Johnson, and J. E. Pratt, "A Controller for the LittleDog Quadruped Walking on Rough Terrain," in IROS-07, 2007.

M. H. Hoepflinger, C. D. Remy, M. Hutter, L. Spinello, and R. Siegwart, "Haptic Terrain Classification for Legged Robots," in ICRA-10, Anchorage, Alaska, 2010, p. in press.

ESA, "Lunar Robotics Challenge (LRC)," 2008, p. http://www.esa.int/esaCP/SEMGAASHKHF_index_0.html.

T. Thueer, P. Lamon, A. Krebs, and R. Y. Siegwart, "CRAB - Exploration Rover with Advanced Obstacle Negotiation Capabilities," in ESA Workshop on Advanced Space Technologies for Robotics, Noordwijk, The Netherlands 2006. 